\title{
Awakening the Analogue Computer: Rubel's Extended Analog Computer Workshop
}

\author{
Jonathan W. Mills \\ School of Informatics and Computing, Indiana University, \\ Bloomington, Indiana, 47404 USA
}

Shannon's General Purpose Analog Computer (GPAC) was unable to directly solve many problems that Lee A. Rubel believed were critical to implement the functions of the human brain. In response Rubel defined the Extended Analog Computer (EAC) in 1993, which was able to directly compute partial differential equations, solve the inverse of functions and implement spatial continuity, among other operations.

Rubel believed that the EAC was an ideal machine, never capable of being built physically. However, research has shown that close approximations to the EAC can be constructed whose functionality is partially contained in the physical structure (implicit computation via the laws of nature), partially contained in the configuration chosen for the material and its energy budget (explicit in the physical computer's structure) and partially, and finally, completed by analogies defined between the EAC and the problem it solves.

This tri-partite solution to problems using the EAC represents a new paradigm for computing, and one that is foreign to most first-time users. During Unconventional Computing 2009 attendees at this workshop will be given a tutorial pamphlet to learn about the EAC, then have the opportunity to conduct experiments on physical EACs available at the conference. They will also learn techniques for the design of "smart matter," that is, applications that run on the EAC supercomputer simulator available at Indiana University.

"Smart matter" as implemented with the EAC is a configuration that is reflexive, limitedly self-aware and self-modifying, and that runs on the large-array hybrid supercomputer simulator sEAC (in the future, our goal is to build a VLSI sEAC supercomputer). A large-array sEAC configuration resembles a proprioceptic cellular automaton that can be configured to solve problems such as protein folding, graph reduction and data mining, among many others. Expressed as a general analogy, smart matter is a kind of "computronium" that allows its users, by a tongue-in-cheek definition of new "elements," to think of their applications as "Proteinium," "NP-Celium," "Dataminium," and other varieties of designed materials.

Techniques for designing smart matter will be presented in the workshop, at a plenary talk at the conference and in Professor Andrew Adamatzky's session on Novel Computing Substrates. 